\title{
Review on Effects of Triphala in Patients with Periodontal Disease
}

\author{
S. Renuka ${ }^{1}$, Priya Lochana G. ${ }^{2}$, Sankari M. ${ }^{3}$
}

\begin{abstract}
${ }^{1}$ Saveetha Dental College, Saveetha Institute of Medical and Technical Sciences (SIMATS), Saveetha University, Chennai, Tamil Nadu, India, ${ }^{2}$ Department of Periodontics, Saveetha Dental College, Saveetha Institute of Medical and Technical Sciences (SIMATS), Saveetha University, Chennai, Tamil Nadu, India, ${ }^{3}$ Department of Periodontics, Saveetha Dental College, Saveetha Institute of Medical and Technical Sciences (SIMATS), Saveetha University, Chennai, Tamil Nadu, India.
\end{abstract}

\section{ABSTRACT}

Periodontal disease is a chronic inflammatory disease which destructs the supporting structures of the tooth and causes tooth loss. Dental plaque is the most common causative agent for periodontal diseases. Based on the location, dental plaque are of two types - supra gingival and sub gingival plaque. Supra gingival plaque is responsible for caries and gingival disease whereas sub gingival plaque is responsible for periodontal destruction. Plaque control can be achieved by combination of mechanical and chemical methods. Mechanical methods aid in removing the irritants during initial phase of treatment, and to maintain the condition, chemical methods like mouthwash, floss and interdental brushes were used. Chlorhexidine is the most commonly used antimicrobial mouthwash for many periodontal conditions and it is known for its substantivity property. Although it has various advantages, prolonged usage of chlorhexidine causes sensitivity of teeth and other side effects. Most of the researches were performed to find an alternative to chlorhexidine mouthwash to overcome its side effects and they believed that herbal mouthwashes with various combinations can be used for prophylactic measures which has no side effects and also act as good anti-microbial agents. Herbal mouthwashes contain phytochemicals that contains anti-microbial and antiinflammatory effect which works without alcohol, artificial preservatives, flavour, or colours. "Triphala" is among the most common formulas used in traditional ayurvedic medicine. It contains fruits of three trees, and they are Indian gooseberry Amalaki (Emblica officinalis), Bibhitaki (Terminalia bellirica), and Haritaki (Terminalia chebula). It has anti-plaque effect due to the presence of tannic acid which adheres to the bacterial cell surface and causes protein denaturation and ultimately to bacterial cell death. Triphala also has antioxidant, anti-inflammatory, anti-collagenase activity. It inhibits the collagenases derived from leukocytes which are responsible for connective tissue destruction in periodontal disease. Hence the usage of Triphala mouthwash treatment of periodontal disease becomes significant. The reason for this review is to give detailed information about the effects of Triphala mouthwash in periodontal disease. Most commonly chlorhexidine mouthwash is used in periodontal therapy even though it has several disadvantages. This review helps in providing effects comparatively similar to chlorhexidine without any adverse effects.

\section{KEY WORDS}

Periodontitis, Triphala, Chlorhexidine, Plaque, Ayurveda
Corresponding Author:

S. Renuka,

No. 162, Poonamallee High Road, Velapanchavadi, Chennai-600077, Chennai-600077, Tamilnadu, India. E-mail: renuka2602@gmail.com

DOI: $10.14260 /$ jemds/2020/215

Financial or Other Competing Interests: None.

How to Cite This Article:

Renuka S, Lochana PG, Sankari M. Review on effects of triphala in patients with periodontal disease. J. Evolution Med. Dent. Sci. 2020;9(12):1000-1005, DOI: $10.14260 /$ jemds/2020/215

Submission 16-01-2020,

Peer Review 27-02-2020,

Acceptance 05-03-2020,

Published 23-03-2020.

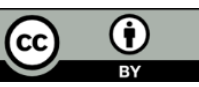




\section{BACKGROUND}

Periodontal disease is a chronic inflammatory disease which destructs the supporting structures of the tooth. The early sign of periodontal disease is initiated in the gingival tissues which aids in the early diagnosis of the disease.[1] Dental plaque is the most common cause for most of the oral diseases such as caries, gingivitis, periodontitis and microbial systemic diseases due to formation of biofilm. ${ }^{[2]}$ Biofilm is the group of microorganisms formed as a matrix of polymers of bacteria over the tooth surface, ${ }^{[3,4]}$ which inhibit the penetration of antimicrobial agents and thus increases the bacterial load in the oral cavity.[5] The initial way of preventing any oral disease is plaque control and prevention of plaque accumulation on the tooth and gingival surface.[6] Dental plaque are of two types- supra gingival and sub gingival plaque based on the location. Supra gingival plaque is responsible for caries and gingival disease whereas sub gingival plaque is responsible for periodontal destruction. Plaque control can be achieved by two ways-mechanical and chemical methods. In mechanical periodontal therapy, scaling is the first recommended treatment for gingivitis and periodontitis ${ }^{[7,8]}$ which helps in removing plaque, particularly sub gingival plaque.

The most common mechanical method of maintaining a good oral hygiene is tooth brushing which aids by removing plaque accumulation. [9] The proximal areas where the tooth brushes are inaccessible that can be cleaned with the help of dental floss. Mechanical plaque control techniques are time consuming and require motivation. Antimicrobial agents such as mouthwashes have been used as an adjunct to mechanical cleaning in initial phase of periodontal therapy as well as in situations where the patient has areas inaccessible to brushing.[10] Many chemical mouthwashes with various compositions are available in the market such as phenolic compounds, Bis-biguanides, pyrimidines, quaternary ammonium compounds, oxygenating agents, halogens, heavy metal salts which has antiseptic and antimicrobial action thus helps in prevention of supra gingival plaque formation that leads to development of gingivitis.[11] Among these Chlorhexidine is the most effective and gold standard mouthwash which helps in plaque inhibition.[12] Though it is the most effectively used mouthwash it cannot be used for long duration as it has many unpleasant side effects which make the herbal mouthwashes popular. Herbal extracts demonstrate immune enhancing, anti-inflammatory, anticancer effects. ${ }^{[13]}$ This review will discuss in detail about the effect of Triphala mouthwash among patients with periodontal disease.

\section{Chlorhexidine Mouthwash}

Chlorhexidine was developed in 1950, which is still considered the most effective mouthwash in dentistry.[14] It is basically antibacterial used as an antiseptic and for other applications. A chemical mouthwash is divided into the first and second generation. The first generation includes phenols, quaternary ammonium compounds and second generation includes bisbiguanides-chlorhexidine gluconate [CHXG] and third generation includes delmopinol.[15] Chlorhexidine mouthwash is effective against both gram positive and gram negative bacteria and it has both bacteriostatic and bactericidal action. ${ }^{[16]}$ It presents immediate bactericidal and extended bacteriostatic action because of adsorption onto the pellicle-lined enamel surface[16] due to the property of substantivity. The overdose of chlorhexidine causes nausea and vomiting, etc. It leads to some side effects such as white patches in mouth or lips, ulcers, swelling of salivary glands, irritation, stains teeth, dry mouth, unpleasant taste, decreased sensation due to an allergic reaction to some individuals.[17] Other chemical mouthwashes are potassium nitrate, hydrogen peroxide, iodide, etc., and it is made of harmful chemical agents in such as thymol, hexetidine, methyl salicylate, eucalyptol, benzalkonium chloride, cetylpyridinium, chloride, methyl paraben, alcohol, and hydrogen peroxide.[17]

\section{Herbal Mouthwash}

Herbs and plant extract have been used in oral hygiene products for many years . In modern medical science, newer drugs are introduced which contains many chemical compounds that exposes the patients to different illness which increases the attention more towards herbal medicines [18]. Herbal medicines are used as it will not cause severe toxicity. Herbal mouthwash contains phytochemical that contains anti-microbial and anti-inflammatory effect which works without alcohol, artificial preservatives, flavour, or colours. clove is traditionally used for oral health in mouthwash because of their antiseptic, antibacterial, and antiviral property, peppermint which gives cooling effect to the mouth, plantain has wound healing capacity and herbs that contains anti-microbial, anti-inflammatory, antioxidants, antiseptic properties such as neem, clove, Triphala with combination of Amalaki, Haritaki, Bibhitaki, tulsi, celery, liquorice, oak tree, bakula, katha, spearmint, turmeric, and Aloe vera.[19] Vegetable glycerine, stevia, and xylitol the sweetener, which foster bacteria and does not allow it to grow. Essential oils used to freshen the breath and also to enhance the strength of the enamel. Many fruit extracts such as pomegranate, guava, cranberry, grapefruit is used as mouthwashes which contains medicinal properties.[17]

\section{Triphala as Mouthwash}

"Triphala" is among the most common formulas used in Ayurvedic medicine. It contains fruits of three trees, and they are Indian gooseberry Amalaki (Emblica officinalis), Bibhitaki (terminalia bellirica), and Haritaki (terminalia chebula).[20] Triphala can be used for dental diseases and most commonly purchased as a bulk powder, tablet, or liquid extract. Triphala is used because of its properties which make its use clinically essential. Following are the properties exhibited by Triphala are free radical scavenging, antibacterial, antimutagenic, wound healing, anticariogenic, antistress, adaptogenic, antioxidant, anti-inflammatory, antipyretic, analgesic, hypoglycaemic, anticancer, chemoprotective, radioprotective and chemopreventive effects.[21] Mehta B K et al (1993) have proved antimicrobial efficacy of Triphala. Triphala because of its components has wide $\mathrm{Gm}+\mathrm{ve}$ and $\mathrm{Gm}$-ve antibacterial activity and antifungal activity.[22] It also shows woundhealing property when applied on wounds externally.[23] It shows complete wound healing even in fully infected dermal wounds. ${ }^{[24]}$ So it can also be used safely in mouth ulcers. Triphala has analgesic, anti-inflammatory effect hence it can use for better maintenance of oral hygiene.[25] According to the study by Shobha tendon, the effect of Triphala extract 
against Streptococcus mutans and Lactobacillus was determined by agar well diffusion method. The zone of inhibition obtained with various concentrations of the extract was observed after $24 \mathrm{~h}$ of incubation at $37^{\circ} \mathrm{C}$. It was found that $0.6 \%$ of Triphala extract showed maximum effect against S. mutans and Lactobacillus. [26] Triphala has anti-collagenases activity which plays a important role in treating periodontal disease. Matrix metalloproteinase play a vital role in periodontal destruction. Doxycycline is most potent tetracycline for collagenases/gelatinase inhibition. However, long-term tetracycline therapy has certain disadvantages. Use of herbal product extract in treating periodontal disease does not produce side-effects of tetracycline compounds as well as other synthetic drugs. Triphala has strong inhibitory action against PMN-type collagenase, especially when MMP-9 exhibits a value of $1500 \mu \mathrm{g} / \mathrm{mL}$ concentration. ${ }^{[27]}$ Triphala has anti-microbial and antioxidant effect against Streptococcus mutans at concentrations as low as $50 \mu \mathrm{g} / \mathrm{mL}$. Triphala has anti-plaque effect due to the presence of tannic acid which adheres to the bacterial cell surface and causes protein denaturation and ultimately to bacterial cell death. The presence of active ingredients of phenolic nature may be responsible for the scavenge of free radicals.[28]

Triphala has the wide spectrum of activity which is used in periodontal therapy as mouthwash. According to the Sushruta Samhita, Triphala can be used as a gargling agent in dental diseases. $0.6 \%$ Triphala mouthwash has shown to have significant anti-caries activity comparable to that of chlorhexidine without any disadvantages as staining of teeth, no evidence of re-mineralization of tooth structure and low cost. Triphala mouth rinse after scaling and root planing showed significant reduction in the plaque, gingival, and oral hygiene indices without any evidence of staining of teeth at seven, 30 , and 45 days, comparative to reduction obtained by chlorhexidine mouth rinse after oral prophylaxis.[29] Triphala mouthwash should be used twice-daily combined with metronidazole $400 \mathrm{mg}$ which is taken thrice-daily, when taken in such a manner results showed improvement in clinical indices in terms of reduction in tooth mobility, pocket depth, bleeding gums, sensitivity to hot and cold, and calculus formation with minimal recurrence in all the clinical parameters comparatively than those exhibited by $0.2 \%$ chlorhexidine twice daily with metronidazole $400 \mathrm{mg}$ thricedaily.[30] The advantage of Triphala mouthwash over chlorhexidine is that it has potential systemic benefits. Triphala has been prescribed for systemic use by Ayurvedic practitioners to cure systemic illnesses. Thus, even if the patient swallows the Triphala mouthwash, it provides systemic benefits. This might prove to be beneficial in disabled patients to maintain their oral hygiene. It has been reported that Triphala is quite safe for use and does not have any side effects and also the ingredients which are used to prepare Triphala are the commonly available fruit derivatives.[31] The Triphala mouthwash can be prepared by 40-50 gm Triphala powder and boil it in $1 \mathrm{ltr}$ water till it reduced to $250 \mathrm{ml}$ and allow it cool down and filter with strainer and keep this decoction in clean sterilized bottle. It can be used three times a day at about 20-50 ml decoction.

\section{Individual Components and Chemicals of Triphala}

The main chemicals constituents found in Triphala are tannins, quinones, flavonoids, vitamin $\mathrm{C}$ and gallic acid.

\section{Tannins}

Are a group of polymeric phenolic substances capable of precipitating gelatin from solution, a property known as astringency. This group of compounds are found in green teas and red wines. Incitement of phagocytic cells, have intervened tumor movement, and an extensive variety of hostile to infective activities, has been doled out to tannins. The counter microbial activity of tannin can inactivate microbial adhesins, compounds, and cell envelope transport proteins. ${ }^{[32]}$

\section{Quinones}

Are important in many biological systems due to its redox potential and it provides a source of stable free radicals, quinones forms irreversible complex with nucleophilic amino acids in proteins, often leading to inactivation of the protein and loss of function. It makes the substrates unsuitable for microorganism. ${ }^{[32]}$

\section{Flavonoids}

Are synthesized by plants in response to microbial infection. Hence it has anti-microbial substances against a wide array of microorganisms analyzed by In-vitro study. It can complex with extracellular and solvable proteins and to complex with bacterial cell dividers. More lipophilic flavonoids disrupt microbial membranes and have been shown resistance against Vibrio cholera 01, Shigella, Streptococcus mutans in vitro. And reduction of fissure caries by about $40 \%$ has also been demonstrated.[32] The only common phytoconstituent present in all three herbs used in Triphala mouth wash is Gallic acid. Gallic acid exhibits hepatoprotective, antioxidant activity and also suppresses growth of cancer cells.[33] Fruit juice of Emblica officinalis (EO) contains the highest vitamin C content. Vitamin C in EO accounts for approximately 45-70\% of the antioxidant activity.[34] Evidences have been reported that significant gum bleeding can occur in vitamin $\mathrm{C}$ deficiency. Vitamin $\mathrm{C}$ along with bioflavonoid aids in the healing process. ${ }^{[35]}$ The components of Triphala are Emblica officinalis, Terminalia chebula and Terminalia bellirica.
Amalaki
Has anti-oxidant and anti-aging properties and it has beneficial role in cancer, diabetes, liver treatment, heart trouble, ulcer, anemia, and various other diseases. Similarly, it has immune modulatory, anti-pyretic, analgesic, cyto protective, anti-tussive, and gastro protective agents. It is also having anti-microbial agent against Escherichia coli, Pseudomonas aeruginosa. Terminalia chebula is a plant species and it is rich in tannin and the main constituents of tannin are chebulic corrosive, chebulagic corrosive, corilagin, and gallic corrosive. Terminalia chebula shows hostile to bacterial movement against various Gram-positive and Gram- negative human pathogenic bacterial species. It likewise shows hostile to contagious and against viral properties. It has additionally demonstrated against mutagenic/hostile to cancer-causing action, cell reinforcement action, adaptogenic and hostile to anaphylactic exercises, immunomodulatory movement, cytoprotective and radioprotective action. It is additionally viable in hypolipidemia/hypercholesterolemia, enhancing gastro-intestinal motility with hostile to uncontrollable movement, diabetes, retinopathy, and wound recuperating. 
Terminalia bellirica is a vast deciduous tree, found all through focal Asia and some different parts of the world. Its organic product is utilized as a part of society pharmaceutical to treat asthma, malignancy, colic, loose bowels, dysuria, cerebral pain, hypertension, aggravations, and torment. The plant contains flavonoids, and tannins. T. bellirica has cancer prevention agent, hostile to bronchodilatory, hypercholesterolemic, against bacterial, cardioprotective, hepatoprotective, hypoglycemic, and hypotensive properties.[36]

\section{Other Effects of Triphala}

Triphala can also be used as a root canal irrigant as it has significant anti-bacterial activity against three and six week biofilms. Primary endodontic infections are caused by number of facultative anaerobic bacteria increases when the root canal remains infected for long periods. The Enterococcus faecalis, a facultative anaerobic gram-positive coccus, is the most common Enterococcus sp. cultured from non-healing endodontic cases. Sodium hypochlorite ( $\mathrm{NaOCl}$ ) is an efficient irrigant used in eliminating E. faecalis biofilms. The use of herbal Triphala as alternatives as a root canal irrigant is because of the several disadvantages of $\mathrm{NaOCl}$ such as unpleasant taste, high toxicity, and its inability to remove the smear layer.[37]

Triphala also has many benefits in systemic illness such as assists nourishes and rejuvenates the tissues, supports healthy digestion and absorption and also act as natural antioxidant. Triphala is most generally accommodating in processing, and supporting normal defecations. The mix of the three natural products has a synergistic impact to support numerous different frameworks also. Notwithstanding the GI tract, Ayurveda utilizes Triphala to help sound respiratory, cardiovascular, urinary, regenerative, and sensory systems [38]. The three organic products engaged with making Triphala has singular impacts which incorporates Amalaki (Emblica officinalis) which as a cooling impact that oversees pitta, supporting the common elements of the liver and the insusceptible framework, Bibhitaki (Terminalia bellirica) bolsters the respiratory framework and in addition kapha gatherings in all frameworks and Haritaki (Terminalia chebula) is still useful for each of the three doshas (vata, pitta, and kapha). Is known for its "scratching" impact, which expels poisons and keeps up sound levels of weight.

\section{Role of Triphala in Periodontitis}

Oxidative stress is an important cause of cell damage associated with the initiation and progression of many chronic diseases. [39-41] Oxidative stress is the disturbance in the pro-oxidant and antioxidant balance resulting in potential tissue damage. Consecutive oxidation-reduction reactions of molecular oxygen by various enzymes results in the production of molecules such as superoxide anion, hydrogen peroxide, hydroxyl radical, singlet oxygen, nitric oxide, hypochlorous acid which together constitute the term "ROS." [42] All the cells in the body are capable of generating ROS, neutrophils are of prime importance with respect to periodontitis, as it is a part of innate immunity which comprise the first line of host defense and are located at sites of microbial invasion. They are activated by inflammatory mediators and can generate increased levels of ROS, which not only attack the periodontopathogens but also the surrounding tissues.[43] The ROS is generated by NADPH oxidase system present in the neutrophils and it catalyses the reduction of molecular oxygen to superoxide anion. Subsequent reductions result in the production of hydrogen peroxide and hydroxyl radical. Similarly, superoxide dismutase enzyme present in all the cells catalyses the dismutation of superoxide radical to hydrogen peroxide. Many studies have shown that ROS regulate the formation and function of osteoclast cells.[44-46] Bone resorption which results in alveolar bone loss and ultimately tooth loss is the hallmark feature of the periodontal disease. NADPH oxidase system plays a role in periodontal pathologies and its involvement is more common in aggressive periodontitis.[47]

Triphala has antimicrobial, antioxidant and anticollagenase properties. The antioxidants present in Triphala reduce the oxidative burden and protect the cells from the damage caused by free radicals.[48,49] Triphala inhibits the collagenases derived from polymorphonuclear leukocytes which are responsible for connective tissue destruction in periodontal disease. Triphala showed $76.6 \%$ reduction of matrix metalloproteinase-9 (MMP-9) activity, whereas kamillosan and doxycycline showed $46.36 \%$ and $58.7 \%$, respectively, at concentrations of $1500 \mu \mathrm{g} / \mathrm{mL}$. Researchers from India's DAPMRV Dental College tested a 6\% Triphala mouthwash demonstrated that the individuals who utilized Triphala mouthwash had a normal $17 \%$ reduction in organisms following 48 hours, and $44 \%$ lessening following seven days. The chlorhexidine group had similar reductions after seven days at about 45\%. Many studies stated that Triphala mouthwash reduced dental decay bacteria similarly to the chlorhexidine mouthwash. In another study from the DAPMRV Dental College, Triphala mouthwash was found to reduce pathogenic bacteria by up to $83 \%$ over a 15 days. Hence Triphala helps in reducing dental decay and periodontal disease comparative to chlorhexidine mouthwash.

\section{CONCLUSIONS}

The aim of this review is to give a detailed description about Triphala mouthwash and its effect on periodontal diseases. Many studies have been conducted to identify the effect of Triphala over standard chlorhexidine mouthwash and stated that Triphala mouthwash reduces the caries and periodontal diseases comparatively similar to chlorhexidine mouthwash, but without any side effects as noticed with chlorhexidine. Dentistry is still in search of herbal drugs to treat the disease that affects the oral cavity. Triphala has the ability to treat the oral diseases without any adverse effects and is also available at minimal cost than commercially available products in the market. This drug has the ability to treat the infectious diseases both locally as well as systemically. It is very safe and effective for the treatment of periodontitis. Many researches have to be encouraged in order to increase the awareness about the pharmacological and clinical effects of Triphala and thereby increase the use of Triphala mouthwash in dentistry. 


\section{REFERENCES}

[1] Desai A, Anil M, Debnath S. A clinical trial to evaluate the effects of Triphala as a mouthwash in comparison with chlorhexidine in chronic generalised periodontitis patient. Indian Journal of Dental Advancements 2010;2(3):243-8.

[2] Narayan A, Mendon C. Comparing the effect of different mouthrinses on de novo plaque formation. J Contemp Dent Pract 2012;13(4):460-3.

[3] Socransky SS, Haffajee AD. Dental biofilms: difficult therapeutic targets. Periodontology 2000 2002;28:1255 .

[4] Marsh PD. Dental plaque as a microbial biofilm. Caries Res 2004;38(3):204-11.

[5] Gilbert P, Maira-Litran T, McBain AJ, et al. The physiology and collective recalcitrance of microbial biofilm communities. Adv Microb Physiol 2002;46:202-56.

[6] Jahangirnezhad M, Amin M, Montazeri AE, et al. In vitro comparison of the effect of shallot extract and chlorhexidine mouthwash on oral pathogens. Afr J Microbol Res 2012;6(6):1262-4.

[7] Theilade E, Wright WH, Jensen SB, et al. Experimental gingivitis in man. II. A longitudinal clinical and bacteriological investigation. J Periodontal Res 1966;1:113.

[8] Cobb CM. Non-surgical pocket therapy: mechanical. Ann Periodontol 1996;1(1):443-90.

[9] Koban I, Holtfreter B, Hubner NO, et al. Antimicrobial efficacy of non-thermal plasma in comparison to chlorhexidine against dental biofilms on titanium discs in vitro - proof of principle experiment. J Clin Periodontol 2011;38(10):956-65.

[10] Lindhe J, Karring T, Lang NP. Clinical periodontology and implant dentistry. Oxford, UK: Blackwell Munksgaard 2003.

[11] Mandel ID. Chemotherapeutic agents for controlling plaque and gingivitis. J Clin Periodontol 1988;15(8):48898,

[12] Ribeiro LG, Hashizume LN, Maltz M. The effect of different formulations of chlorhexidine in reducing levels of mutans streptococci in the oral cavity: a systematic review of the literature. J Dent 2007;35(5):359-70.

[13] Rao NJ, Subhas KR, Kumar KS. Role of phytotherapy in gingivitis; a review. Int J Pharmacol 2012;8(1):1-5.

[14] Aspalli S, Shetty VS, Devarathnamma MV, et al. Evaluation of antiplaque and antigingivitis effect of herbal mouthwash in treatment of plaque induced gingivitis: a randomized, clinical trial. J Indian Soc Periodontol 2014;18(1):48-52.

[15] Mhaske M, Samad BN, Jawade R, et al. Chemical agents in control of dental plaque in dentistry: an overview of current knowledge and future challenges. Adv Appl Sci Res 2012;3(1):268-72.

[16] Jenkins S, Addy M, Wade W. The mechanism of action of chlorhexidine. A study of plaque growth on enamel inserts in vivo. J Clin Periodontol 1988;15(7):415-24.

[17] Renuka S, Muralidharan NP. Comparison in benefits of herbal mouthwashes with chlorhexidine mouthwash: a review. Asian J Pharm Clin Res 2017;10(2):3-7.
[18] Maurya DK, Mittal N, Sharma KR, et al. Role of triphala in the management of peridontal disease. Ancient Science of Life 1997;17(2):120-7.

[19] Biswas G, Anup N, Acharya S, et al. Evaluation of the efficacy of $0.2 \%$ chlorhexidine versus herbal oral rinse on plaque induced gingivitis - a randomized clinical trial. IOSR J Nurs \& Health Sci (IOSR-JNHS) 2014;3(2):58-63.

[20] Bajaj N, Tandon S. The effect of Triphala and Chlorhexidine mouthwash on dental plaque, gingival inflammation, and microbial growth. International Journal of Ayurveda Research 2011;2(1):29-36.

[21] Baliga MS, Meera S, Mathai B, et al. Scientific validation of the ethnomedicinal properties of the Ayurvedic drug Triphala: a review. Chinese Journal of Integrative Medicine 2012;18(12):946-54.

[22] Chattopadhyay RR, Bhattacharyya SK. Plant review: Terminalia chebula: an update. In Pharmacognosy Reviews 2007;1(1):151-6.

[23] Kumar MS, Kirubanandan S, Sripriya R, et al. Triphala promotes healing of infected full-thickness dermal wound. J Surgical Res 2008;144(1):94-101.

[24] Kumar MS, Kirubanandan S, Sripriya R, et al. Triphala incorporated collagen sponge: a smart biomaterial for infected dermal wound healing. J Surgical Res 2010;158(1):162-70.

[25] Prabu D, Kirubanandan S, Ponnudurai K, et al. Preliminary evaluation of anti-inflammatory and analgesic activities of triphala. Int J Nat and App Sci 2007;3(4):419-24.

[26] Tandon S, Gupta K, Rao S, et al. Effect of Triphala mouthwash on the caries status. Int J Ayurveda Res 2010;1(2):93-9.

[27] Prabhakar J, Senthilkumar M, Priya MS, et al. Evaluation of antimicrobial efficacy of herbal alternatives (Triphala and Green Tea polyphenols), MTAD and 5\% Sodium Hypochlorite against Enterococcus faecalis biofilm formed on tooth substrate: an in vitro study. J Endod 2010;36(1):83-6.

[28] Jagdish L, AnandKumar VK, Kaviyarasan V. Effect of Triphala on dental biofilm. Indian J Sci Technol 2009;2:30-3.

[29] Desai A, Anil M, Debnath S. A clinical trial to evaluate the effects of Triphala as a mouthwash in comparison with chlorhexidine in chronic generalized periodontitis patient. Indian J Dent Adv 2010;2(3):243-7.

[30] Maurya DK, Mittal N, Sharma KR, et al. Role of triphala in the management of periodontal disease. Anc Sci Life 1997;17(2):120-7.

[31] Srinagesh J, Krishnappa P, Somanna SN. Antibacterial efficacy of triphala against oral streptococci: an in vivo study. Indian Journal of Dental Research 2012;23(5):696.

[32] Cowan MM. Plant products as antimicrobial agents. Clinical Microbiology Reviews 1999;12(4):564-82.

[33] Mukherjee PK, Rai S, Bhattacharyya S, et al. Clinical study of 'Triphala'- a well-known phytomedicine from India. Iran J Pharmacol Ther 2006;5:51-4.

[34] Khan KH. Roles of Emblica officinalis in medicine: a review. Bot Res Int 2009;2:218-28.

[35] Desai A, Anil M, Debnath S. A clinical trial to evaluate the effects of Triphala as a mouthwash in comparison with 
chlorhexidine in chronic generalized periodontitis patient. Indian J Dent Adv 2010;2:243-7.

[36] Khan AU, Gilani AH. Antisecretory and analgesic activities of Terminalia bellerica. Afr J Biotechnol 2010;9(18):27179.

[37] Abraham S, Kumar MS, Sehgal PK, et al. Evaluation of the inhibitory effect of Triphala on PMN-type Matrix Metalloproteinase (MMP-9). J Periodontol 2005;76(4):497-502.

[38] Pole, Sebastian. Ayurvedic medicine: the principles of traditional practice. Churchill Livingston 2006: p. 126-7, 145-6, 194-5.

[39] Matthews AT, Ross MK. Oxyradical stress, endocannabinoids and atherosclerosis. Toxics 2015;3(4):481-98.

[40] Fischer BM, Voynow JA, Ghio AJ. COPD: Balancing oxidants and antioxidants. Int J Chron Obstruct Pulmon Dis 2015;10:261-76.

[41] D'Aiuto F, Nibali L, Parkar M, et al. Oxidative stress, systemic inflammation, and severe periodontitis. J Dent Res 2010;89(11):1241-6.

[42] Devasagayam TPA, Tilak JC, Boloor KK, et al. Free radicals and antioxidants in human health: current status and future prospects. J Assoc Physicians India 2004;52:794804.
[43] Nagata M. Inflammatory cells and oxygen radicals. Curr Drug Targets Inflamm Allergy 2005;4(4):503-4.

[44] Garrett IR, Boyce BF, Oreffo RO, et al. Oxygen-derived free radicals stimulate osteoclastic bone resorption in rodent bone in vitro and in vivo. J Clin Invest 1990;85(3):632-9.

[45] Bax BE, Alam AS, Banerji B, et al. Stimulation of osteoclastic bone resorption by hydrogen peroxide. Biochem Biophys Res Commun 1992;183(3):1153-8.

[46] Lean JM, Jagger CJ, Kirstein B, et al. Hydrogen peroxide is essential for estrogen-deficiency bone loss and osteoclast formation. Endocrinology 2005;146(2):728-35.

[47] Giannopoulou C, Krause KH, Müller F. The NADPH oxidase NOX2 plays a role in periodontal pathologies. Semin Immunopathol 2008;30(3):273-8.

[48] Vani T, Rajani M, Sarkar S, et al. Antioxidant properties of the ayurvedic formulation triphala and its constituents. Int J Pharmacog 1997;35(5):313-7.

[49] Padmawar A, Bhadoriya U. Phytochemical investigation and comparative evaluation of in vitro free radical scavenging activity of triphala and curcumin. Asian J Pharm Med Sci 2011;1:9-12. 